\title{
Bedarf für eine palliativmedizinische Versorgung in der Pneumologie bei nicht malignen Erkrankungen - ein Fallbericht
}

\author{
The Need for Palliative Care Treatment in Pneumology in Non-Malignant \\ Diseases - A Case Report
}

Autoren

Institute
W. Nehls ${ }^{1}$, S. Delis ${ }^{1}$, N. Black' ${ }^{1}$ S. Gabrijel ${ }^{1}$, H. Albrecht ${ }^{2}$, T. T. Bauer ${ }^{1}$

${ }^{1}$ Lungenklinik Heckeshorn, Klinik für Pneumologie, HELIOS Klinikum Emil von Behring, Berlin

(Leiter: Prof. Dr. med. Torsten T. Bauer)

${ }^{2}$ Klinik für Psychosomatische Medizin und Psychotherapie (Leiter: Dr. med. Dipl.-Psych. Helmut Albrecht) $\begin{array}{lll}\text { eingereicht } & \text { 4. 3. } 2012\end{array}$ akzeptiert nach Revision 12. 3.2012

\section{Bibliografie}

Dol http://dx.doi.org/ 10.1055/s-0032-1308913 Pneumologie 2012; 66: 231-234

(c) Georg Thieme Verlag KG Stuttgart · New York ISSN 0934-8387

\section{Korrespondenzadresse} Wiebke Nehls

Lungenklinik Heckeshorn Klinik für Pneumologie HELIOS Klinikum Emil von Behring Walterhöferstr. 11 14164 Berlin wiebke.nehls@helios-kliniken.de

\section{Zusammenfassung \\ $\nabla$}

Hintergrund: Ein 70-jähriger pneumologischer Patient mit seit vielen Jahren bestehender COPD und Sauerstofflangzeittherapie entwickelte im Verlauf seiner Erkrankung ein komplexes Beschwerdebild. Im mehrwöchigen stationären Verlauf erfolgte eine Behandlung von ausgeprägten körperlichen Symptomen wie Luftnot, Angst und Schmerzen. Nach Übernahme auf die Palliativstation war schließlich nur noch die umfassende finale Begleitung im stationären Setting möglich. Die in unserem Zentrum vorhandene palliativmedizinische Expertise wurde in diesem Fall zu spät in die therapeutischen Prozesse mit einbezogen. Das führte zu einer schlechteren Kontrolle der oben genannten Symptome. Des Weiteren konnte der Wunsch des Patienten nach umfassender ambulanter Versorgung nicht rechtzeitig umgesetzt werden, da die Schwere des Krankheitsbildes nicht frühzeitig erfasst wurde. Instrumente zur rechtzeitigen Erfassung eines nicht malignen pneumologischen Krankheitsbildes mit Bedarf einer zusätzlichen palliativmedizinischen Versorgung sind nicht etabliert.

\section{Fallbeschreibung}

$\nabla$

Anamnese: Wir übernahmen den Patienten im Oktober 2010 aus einem anderen Krankenhaus, wo er bereits wegen einer schweren Exazerbation der chronisch-obstruktiven Lungenerkrankung (AE-COPD, Typ II nach Stockley) aufgenommen und behandelt worden war. Seit mehreren Monaten wurde im häuslichen Bereich eine Sauerstofflangzeittherapie durchgeführt. Der letzte stationäre Aufenthalt in unserer Klinik lag 4 Monate zurück. Es bestand eine ausgeprägte kardiovaskuläre Komorbidität mit absoluter Arrhythmie bei Vorhofflimmern und Rechtsherzinsuffizienz, eine Osteoporose mit Deckplatteneinbruch LWK 2-3 und Spondylarthrose, Zustand nach Schulter-TEP

\section{Abstract \\ $\nabla$}

Background: A 70-year-old pneumological patient with a COLD for many years, who was using long-term oxygen therapy, developed a complex clinical presentation. During his inpatient course over several weeks we treated severe physical symptoms such as shortness of breath, anxiety and pain. After the transfer to the palliative care ward only an inpatient final accompaniment was possible. In this case the palliative care expertise was integrated too late into the process of the therapy. The delay led to a poorer control of severe physical symptoms such as those named above. Also, the patients request for comprehensive ambulant care could not be realised because the severity of the disease was not detected soon enough. Instruments for the evaluation of the need for an additional palliative care treatment in patients with a non-malignant pneumological disease have not been established sufficiently.

linksseitig 01/2007 bei Fraktur mit postoperativer Bewegungseinschränkung, eine chronische Niereninsuffizienz und ein Zustand nach Apoplex mit Amaurosis fugax 2003.

Körperliche Untersuchung: Patient in deutlich reduziertem Allgemeinzustand und adipösem Ernährungszustand (70 Jahre, $165 \mathrm{~cm}, 89 \mathrm{~kg} \mathrm{KG}$, BMI 32,7). Die Atemfrequenz betrug 29/min mit ausgeprägtem Giemen und Brummen über der gesamten Lunge. Es bestanden eine geringe Lippenzyanose sowie erhebliche Unterschenkelödeme beidseits. 


\section{Lungenfunktionsprüfung und Blutgasanalyse}

\begin{tabular}{|lll|}
\hline \multicolumn{2}{ll}{ Lungenfunktionswerte } \\
\hline VCmax & $1,88 I$ & $(44,0 \%$ vom Soll $)$ \\
\hline FEV1 & $0,95 I$ & $(30,0 \%$ vom Soll $)$ \\
\hline FEV1\%VCmax & $50,86 \%$ & $(67,7 \%$ vom Soll $)$ \\
\hline FVC & $1,65 I$ & $(40,6 \%$ vom Soll $)$ \\
\hline RV & $4,31 I$ & $(169 \%$ vom Soll $)$ \\
\hline RV/TLC & $69,69 \%$ & $(173,8 \%$ vom Soll $)$ \\
\hline TLC & $6,19 I$ & $(88,6 \%$ vom Soll $)$ \\
\hline Rtot & $0,72 \mathrm{kPa} \times \mathrm{s} / \mathrm{I}$ & $($ normal $<0,35 \mathrm{kPa} \times \mathrm{s} / \mathrm{l})$ \\
\hline
\end{tabular}

Kapilläre Blutgasanalyse unter Raumluft:

pH 7,49; $\mathrm{PAO}_{2} 59,2 \mathrm{mmHg} ; \mathrm{PaCO}_{2} 40 \mathrm{mmHg} ; \mathrm{SO}_{2} 92,3 \%$.

Kapilläre Blutgasanalyse mit Sauerstoffzufuhr von 2 Litern/min: pH 7,50; $\mathrm{PaO}_{2} 69,7 \mathrm{mmHg} ; \mathrm{PaCO}_{2} 38,4$ mmHg; $\mathrm{SO}_{2}$ 95,8\%.

\section{Röntgen-Thorax-Aufnahme in 2 Ebenen}

Streifige Zeichnungsvermehrung parakardial beidseits wie bei chronischer Bronchitis, kein Nachweis konfluierender Infiltrate. Kein Pleuraerguss. Bilateral gering verbreitertes Herz, keine Zeichen der kardialen Dekompensation. Kein Pneumothorax. Degenerative Veränderungen der BWS. Proximale Humerusendoprothese links.

\section{Bakteriologische Untersuchung von Sputum}

Massenhaft Nachweis von Streptococcus pneumoniae, sensibel auf z.B. Ampicillin.

\section{Verlauf}

Bei Exazerbation der COPD (GOLD IV) Typ II nach Stockley mit dekompensierter Rechtsherzinsuffizienz erfolgte eine Behandlung mit optimierter inhalativer Therapie über IPPB, parenteraler Steroidgabe, resistenzgerechter antibiotischer Therapie und diuretischer Therapie. Der Allgemeinzustand des Patienten besserte sich nur geringfügig. Häufig traten Dyspnoeattacken bei erheblicher bronchialer Spastik, vor allem in den Nachtstunden und an Wochenenden, auf. Es erfolgte zusätzlich eine bedarfsgerechte subkutane Morphingabe bei anfallsartiger Luftnot (5 mg MSI s.c.), diese wurde von dem Pat. kaum genutzt.

Aufgrund von ausgeprägten Schmerzen (Numerische Rating-Skala (NRS) - Schmerz 7 - 9) bei bekannter Osteoporose und Wirbelkörperfraktur begannen wir eine Schmerztherapie mit transdermalen Opioiden, die Dosis wurde sukzessive erhöht (Fentanyl $100 \mu \mathrm{g} / \mathrm{h}$ ). Eine Mobilisation war aufgrund von Dyspnoe und Schmerzen nur bedingt möglich. Aufgrund der ausgeprägten Atemnot konnte der Patient nur kurze Wegstrecken (unter 20 Metern) zurücklegen.

In der weiteren Planung einer Entlassung kamen im Gespräch mit dem Patienten eine depressive Grundhaltung und Ängste aufgrund der zunehmenden Hilflosigkeit hervor. Der Patient war Bankkaufmann im Ruhestand, in vierter Ehe verheiratet, ohne Kinder und lebte mit seiner Ehefrau zusammen. Ein Stiefsohn ist vor 7 Jahren mit 43 Jahren an einem Lungenkarzinom verstorben, es bestand ein sehr guter Kontakt zu der ehemaligen Schwiegertochter. Als Interessen gab der Patient das Sammeln von Münzen und Briefmarken sowie das Lösen von Kreuzworträtseln an. Einen Umzug in eine stationäre Pflegeeinrichtung lehnte der Patient ab, ebenso die Inanspruchnahme eines ambulanten Pflegedienstes. Er äußerte mehrfach den Wunsch, vor seiner Frau zu sterben, um ihr nicht zur Last zu fallen.
Aufgrund der hohen Symptomlast des Patienten bei schwergradiger chronisch-obstruktiver Lungenerkrankung nahmen wir kurz vor geplanter Entlassung Kontakt zu unserem palliativmedizinischen Konsildienst auf. In einem einmaligen Kontakt erfolgte eine Therapieempfehlung zur Optimierung der anxiolytischen und analgetischen Therapie sowie eine Information über die Möglichkeiten der stationären und ambulanten Palliativversorgung. Nach insgesamt 54 Tagen Liegedauer in unserer Klinik wurde der Patient nach Hause entlassen.

Bereits vier Tage später stellte sich der Patient mit zunehmender Dyspnoe sowie Allgemeinzustandsverschlechterung in der Notaufnahme unseres Hauses vor. Daraufhin wurde der Patient kurzzeitig auf die pneumologische Station aufgenommen und konnte bereits einen Tag später auf die Palliativstation zu weiteren supportiven Maßnahmen verlegt werden. Es bestanden neben der deutlichen Dyspnoe-Symptomatik Schmerzen am gesamten Rücken sowie an beiden Schultern (NRS 5-7 - mixed pain), Husten ohne Auswurf und körperliche Schwäche. Weiterhin bestand eine erhebliche Belastung im psychosozialen Bereich aufgrund der fortschreitenden Erkrankung und der ungewissen weiteren Versorgung im ambulanten Bereich. Im stationären Verlauf wurde der Patient selten durch seine Ehefrau besucht, sodass hier kaum gemeinsame Gespräche möglich waren, die zur Entlastung der Situation geführt hätten. Uns war bekannt, dass die Ehefrau ebenfalls körperlich durch verschiedene internistische Vorerkrankungen eingeschränkt war. Zur Erleichterung der Dyspnoe sowie zur Linderung der deutlichen Schmerzsymptomatik wurde die transdermale Therapie mit Fentanyl auf eine orale Medikation mit Oxycodon umgestellt und bedarfsgerecht gesteigert (Tagesdosis: $2 \times 120 \mathrm{mg}$ ). Der Patient wurde noch einmal ausführlich über den Sinn einer Notfallmedikation mit Opioiden zur Linderung von Schmerz und Dyspnoe informiert. Schnell wirksame Opioide wurden nun regelmäßig von ihm genutzt. Durch physikalische Therapie konnten die Schmerzen an beiden Schultern zusätzlich gelindert werden. Zur Besserung der Krankheitsverarbeitung und zur Linderung der reaktiven Depression profitierte der Patient von den regelmäßigen Gesprächen mit der Mitarbeiterin der psychosomatischen Abteilung, in denen seine Angst und seine Einsamkeit thematisiert wurden. Er zeigte im Verlauf erstmals eine emotionale Entlastung und suchte im stationären Verlauf einen offenen und intensiven Kontakt zu unserem evangelischen Seelsorger.

Die Versorgung zu Hause wurde bis zur Aufnahme ins Krankenhaus durch die Frau des Patienten unterstützt, die selber in körperlich reduziertem Allgemeinzustand war. Die Unterstützung durch einen Pflegedienst wurde von dem Patienten bisher nicht gewünscht. Da der Patient weiterhin den Wunsch äußerte zu Hause versorgt zu werden, wurden Kontakte zur spezialisierten ambulanten Palliativversorgung (SAPV) hergestellt. Mit einer zusätzlichen Unterstützung durch einen Pflegedienst war der Patient nun einverstanden.

Trotz Intensivierung der medikamentösen Therapie nahmen die Knöchelödeme, die bronchiale Hypersekretion und die Dyspnoe weiter zu, sodass von der geplanten Entlassung nach Hause Abstand genommen werden musste. Bei progressiver Allgemeinzustandsverschlechterung wurde der Patient bis zu seinem Tod am 13.01.11 von seiner Frau und seiner Schwiegertochter zusammen mit unserem Palliativteam eng begleitet und die Familienangehörigen im Abschied unterstützt. 


\section{Diskussion}

\section{$\nabla$}

Der beschriebene Fall zeigt, dass der Bedarf an strukturierter palliativmedizinischer Versorgung bei Patienten mit COPD aufgrund des regelhaft phasenförmigen Verlaufs mit Exazerbation und anschließender Besserung schwer einzuschätzen ist. In der Pneumologie bestehen bei malignen Grunderkrankungen inzwischen gute Erfahrungen mit der zeitgerechten Implementierung der Palliativmedizin. So konnte gezeigt werden, dass Patienten mit einem nicht-kleinzelligen Lungenkarzinom von der zusätzlichen frühzeitigen palliativmedizinischen Versorgung hinsichtlich der Lebensqualität deutlich profitierten und darüber hinaus sogar in dieser Studie eine verlängerte Lebenszeit (11,6 Monate vs. 8,9 Monate) aufwiesen [1]. Die bedarfsgerechte Integration der Palliativmedizin bei nicht malignen Krankheitsbildern, wie die in der Kasuistik beschriebene COPD, aber z.B. auch bei Patienten mit Lungenfibrosen, ist schwieriger zu etablieren. Aufgabe einer Palliativstation eines Lungenzentrums ist es, Patienten mit fortgeschrittenen pneumologischen Erkrankungen und komplexer Symptomlast zu behandeln. Hier ist es eine besondere Herausforderung, die Patienten mit einer fortgeschrittenen COPD bereits vor Erreichen der Terminalphase zu erkennen, auch wenn das Sterben weder absehbar noch thematisiert ist.

Patienten mit einer fortgeschrittenen COPD leiden unter einer ähnlich starken Symptomlast wie Patienten mit Lungenkarzinomen [2]. Neben der im Vordergrund stehenden Dyspnoe und Husten bestehen häufig starke Schmerzen, Ängste und Depression. Zudem berichten die Patienten über Fatigue, Schlafstörungen, aber auch Mundtrockenheit, Obstipation, Anorexie oder körperliche Schwäche [3].

Zu Beginn der Behandlung von Dyspnoe steht ein ausführliches Assessment. Die Evidenz für die medikamentöse Therapie der Dyspnoe mit Opioiden ist bei COPD-Patienten gut [4,5]. Darüber hinaus stehen zahlreiche validierte nichtmedikamentöse Methoden [6] wie Atemkontrolltechniken, Entspannungsmaßnahmen (progressive Muskelrelaxation und Ablenkungsübungen) sowie Reflexion des Zusammenhangs der Erkrankung, der Atemnot und der eigenen Zukunftsperspektive zur Verfügung [7]. Durch den Einsatz von Hilfsmitteln wie Rollatoren zur Unterstützung der Atemhilfsmuskulatur [8] und Handventilatoren kann die Atemnot zusätzlich positiv beeinflusst werden [9].

Gleiches gilt für die Therapie von Schmerzen, auch hier steht die Erfassung des Ausmaßes an erster Stelle. Ein grundlegendes Prinzip der Therapie chronischer Schmerzen ist die Bevorzugung einer oralen Applikation vor einer transdermalen Gabe [10]. Die Opioidtherapie ist auch bei Patienten mit chronischen Schmerzen, die nicht auf eine maligne Erkrankung zurückzuführen sind, etabliert [11]. Bei starken Schmerzen mit deutlichen tageszeitlichen Schwankungen - wie in unserer Kasuistik beschrieben sind orale Medikamente besser steuerbar. Weiterhin ist die Pharmakokinetik einer transdermalen Opioidgabe bei Patienten mit Schweißneigung und Kachexie unsicher, sodass wir die Opioidrotation auf ein orales Präparat erfolgreich umsetzen konnten.

Gore et al. konnten in ihrer Studie zeigen, dass klinisch relevante Angst und/oder Depressionen ein mit 90\% Auftreten sehr häufiges - aber oft auch unbeachtetes - Symptom ist. Bemerkenswert ist, dass im Vergleich dazu nur 52\% der Lungenkarzinom-Patienten unter Angst bzw. Depressionen litten [3]. Aufgrund der häufig bestehenden psychosozialen Belastung der COPD-Patienten ist die Einbeziehung der Psychotherapie und der Seelsorge wünschenswert.
Die palliativmedizinische stationäre Betreuung hatte sich auch in der Vergangenheit definitionsgemäß nicht auf Patienten mit Krebserkrankungen beschränkt. Dennoch profitierten von diesem umfassenden Ansatz bisher fast ausschließlich onkologische Patienten, die mit ca. 98\% auf den Stationen überrepräsentiert waren [12]. Der Gesetzgeber führte 2007 die Spezialisierte Ambulante Palliativversorgung (SAPV) in das Sozialgesetzbuch V ein [13]. Ziel der veränderten Gesetzeslage war es, schwerkranken und stark symptombelasteten Palliativpatienten zu ermöglichen, bis zu ihrem Tod in ihrer gewohnten Umgebung versorgt zu werden und Krankenhauseinweisungen am Lebensende zu vermeiden. Bisher kam die SAPV überwiegend Tumorpatienten zugute [14]. Der Anteil der Patienten, die eine intensive ambulante Betreuung im Rahmen einer SAPV benötigen, wird auf 5\% aller an nicht tumorbedingten Erkrankungen in der Terminalphase Leidenden geschätzt [15]. Diese Schätzung ist aus unserer Sicht vermutlich zu gering; der reelle Bedarf an SAPV-Betreuung bei nicht malignen Erkrankungen liegt wahrscheinlich weitaus höher. Inzwischen sind in allen Bundesländern Rahmenverträge zwischen den Kostenträgern und Leistungserbringern geschlossen worden, die sich zum Teil deutlich voneinander unterscheiden. Die Berliner Situation weicht von andern Bundesländern ab; wir blicken auf eine langjährige palliativärztliche Erfahrung mit der ambulanten Versorgung von onkologischen Palliativpatienten zurück. Home Care Berlin e. V. (HCB) bot eine ärztliche Palliativversorgung mit gesonderter Vergütung seit 1994 für Patienten an. Der Rahmenvertrag in Berlin über die spezialisierte ambulante Palliativversorgung vom 01.07.2010 zwischen der Kassenärztlichen Vereinigung, HCB und den gesetzlichen Krankenkassen [16] erweitert nun den Kreis der Anspruchsberechtigten auch auf Patienten mit nicht malignen, unheilbaren und fortgeschrittenen Erkrankungen, die mit einem komplexen Beschwerdebild einhergehen. Damit werden die zum Teil jahrelang ambulant tätigen Palliativmediziner mit neuen Diagnosen wie der COPD konfrontiert.

Eine Möglichkeit einer frühzeitigen und vorausschauenden Versorgungsplanung ist das sogenannte „Advance Care Planning“, welches eine Strategie beschreibt, die weit über den Inhalt einer Patientenverfügung hinaus geht. „Advance Care Planning“ ist vor allem im englischen Sprachraum verbreitet. Dieser Prozess, der sich als kontinuierlicher Dialog zwischen Behandler und Patient versteht, hilft den Patienten und ihren Familien, Werte und Ziele in Bezug auf ihre Gesundheit und einer medizinischen Behandlung zu erkennen und vorauszuplanen [17]. Bei COPD-Patienten beinhalten diese Gespräche unter anderem eine umfassende Information von Patient und Angehörigen zu den Themen Prognose, Möglichkeiten einer invasiven Therapie wie maschineller Beatmung mit ihren Grenzen und auch das Besprechen der finalen Erkrankungsphase.

Im Vergleich zu Patienten mit Lungenkrebs versterben Patienten mit einer COPD eher im Krankenhaus [18]. Mit besserer palliativmedizinischer Versorgung dieser COPD-Patienten können wir der Tatsache, dass 75\% der Palliativpatienten zu Hause versterben wollen, in der Zukunft eher gerecht werden [19].

End-of-life-decision-making sollte gemeinsam mit Patienten, Familie und Medizinern erfolgen. Dabei sind Lebensqualität, Ziele und Wünsche des Patienten zu berücksichtigen [20]. Zahlreiche Untersuchungen belegen, dass Patienten mit lebensbegrenzenden Erkrankungen von einer offenen Kommunikation über Endof-Life-Themen profitieren [21,22]. 
Im Rahmen der intensiven Vernetzung der Pneumologie und Palliativmedizin sowohl in der ambulanten als auch stationären Versorgung mit bedarfsgerechten therapeutischen Ansätzen sollte eine spezialisierte pneumologisch-palliativmedizinische Versorgungstruktur entstehen.

\section{Interessenkonflikt}

$\nabla$

Die Autoren geben an, dass kein Interessenkonflikt besteht.

\section{Literatur}

1 Temel J, Greer J, Muzikansky A et al. Early Palliative Care for Patients with Metastatic Non-Small-Cell Lung Cancer. N Engl J Med 2010; 363: $733-742$

2 Gore JM, Brophy CJ, Greenston MA. How well do we care for patients with end stage chronic obstructive pulmonary disease (COPD)? A comparison of palliative care and quality of life in COPD and lung cancer. Thorax 2000; 55: 1000 - 1006

3 Conventry PA, Grande GE, Richards DA et al. Prediction of appropriate timing of palliative care for older adults with nonmalignant life-threatening disease: a systematic review. Age Aging 2005; 34: 218-227

4 Jennings AL, Davies AN, Higgins JP et al. A systematic review of the use of opioids in the management of dyspnoea. Thorax 2002; 57: 939-944

5 Abernethy AP, Currow DC, Frith $P$ et al. Randomised, double blind, placebo controlled crossover trial of sustained release morphine for the management of refractory dyspnoea. BMJ 2003; 327: 523-528

6 Bausewein C, Booth S, Gysels M et al. Non-pharmacological interventions for breathlessness in advanced stages of malignant and non-malignant diseases. Cochrane Database Syst Rev 2008; 2: CD005623

7 Bredin M, Corner J, Krishnasamy $M$ et al. Multicentre randomised controlled trial of nursing intervention for breathlessness in patients with lung cancer. BMJ 1999; 318: 901

8 Marciniuk DD, Goodridge D, Hernandez $P$ et al. Managing dyspnea in patients with advanced chronic obstructive pulmonary disease: a Canadian Thoracic Society clinical practice guideline. Can Respir J 2011; 18: $69-78$

9 Galbraith S, Fagan P, Perkins $P$ et al. Does the use of a handheld fan improve chronic dyspnea? A randomized, controlled, crossover trial. J Pain Symptom Manage 2010; 39: 831 -838
10 WHO. Cancer Pain Relief. With a guide to opioid availablility. Geneva: WHO; 1996; 2nd edition; 36-37

11 Dertwinkel R, Wiebalck A, Zenz M et al. Oral opioids for long-term treatment of chronic non-cancer pain. Anästhesist 1996; 45: 495-505

12 Radbruch L, Ostgathe C, Elsner F et al. What are the problems in palliative care? Results from a representative survey. Support Care Cancer 2003; 11: $442-451$

13 Bundesministerium d. Justiz. Sozialgesetzbuch V§37b. Gesetzliche Krankenversicherung. 2007. Abgerufen am 18. Januar 2012: http:// www.gesetze-im-internet.de/sgb_5/_37b.html

14 Lindena G, Woskanjan S, Fahrland R. HOPE-Gesamtbericht. 2010. Abgerufen am 21. Januar 2012 https://www.hope-clara.de/download/ HOPE_2010

15 Nordrhein L-Ä. Rahmenprogramm zur flächendeckenden Umsetzung der ambulanten palliativmedizinischen und palliativpflegerischen Versorgung in NRW - kooperatives integratives Versorgungskonzept. 2006. Abgerufen am 18. Januar 2012 http://www.mags.nrw.de/ 08_PDF/002/konzept-palliativ.pdf

16 KV Berlin, Home Care Berlin, gesetzliche Krankenkassen. Rahmenvertrag über die spezialisierte ambulante Palliativversorgung gemäß $\S 132 d$ SGB V in Berlin. 2010. Abgerufen am 18. Januar 2012 http:// www.kvberlin.de/20praxis/60vertrag/10vertraege/sapv/ sapv_100701.pdf

17 Emanuel L, von Gunten C, Ferris FD. Advance Care Planning. Arch Fam Med 2000; 10: 1181 - 1887

18 Edmonds P, Karlsen S, Khan $S$ et al. A comparison of the palliative care needs of patients dying from chronic respiratory diseases and lung cancer. Pall Med 2001; 04: 287-295

19 Oorschot O, Hausmann C, Köhler $N$ et al. Patienten als Partner in der letzten Lebensphase. Bundesgesundheitsbl - Gesundheitsforsch - Gesundheitsschutz 2001; 47: 992 - 999

20 Rhodes P. Palliative Care: the situation of people with chronic respiratory disease. J Am Geriatr Soc 1999; 48: 91 - 100

21 Wright A, Zhang B, Ray A et al. Association between end-of-life discussions, patient mental health, medical care near death, and caregiver bereavement adjustment. JAMA 2008; 300: 1665-1673

22 Reinke L, Slatore C, Uman J et al. Patient-Clinician Communication about End-of-Life Care Topics: Is Anyone Talking to Patients with Chronic Obstructive Pulmonary Disease? J Palliat Med 2001; 14: 923-928 